\title{
Building Cancer Prevention and Control Research Capacity in Rural Appalachian Kentucky Primary Care Clinics During COVID-19: Development and Adaptation of a Multilevel Colorectal Cancer Screening Project
}

\author{
Aaron J. Kruse-Diehr ${ }^{1,2}$ (D) $\cdot$ Mark Dignan $^{2,3} \cdot$ Mark Cromo $^{3} \cdot$ Angela L. Carman $^{1} \cdot$ Melinda Rogers $^{2} \cdot$ David Gross $^{4} \cdot$ \\ Sue Russell ${ }^{4}$
}

Accepted: 7 February 2021/Published online: 18 February 2021

(C) American Association for Cancer Education 2021

\begin{abstract}
This study describes the development of a colorectal cancer (CRC) screening multilevel intervention with four primary care clinics in rural Appalachian Kentucky. We also discuss barriers experienced by the clinics during COVID-19 and how clinic limitations and needs informed project modifications. Four primary care clinics were recruited, key informant interviews with clinic providers were conducted, electronic health record (EHR) capacity to collect data related to CRC screening and follow-up was assessed, and a series of meetings were held with clinic champions to discuss implementation of strategies to impact clinic CRC screening rates. Analysis of interviews revealed multilevel barriers to CRC screening. Patient-level barriers included fatalism, competing priorities, and financial and literacy concerns. The main provider- and clinic-level barriers were provider preference for colonoscopy over stool-based testing and EHR tracking concerns. Clinics selected strategies to address barriers, but the onset of COVID-19 necessitated modifications to these strategies. Due to COVID-19, changes in clinic staffing and workflow occurred, including provider furloughs, a state-mandated pause in elective procedures, and an increase in telehealth. Clinics adapted screening strategies to match changing needs, including shifting from paper to digital educational tools and using telehealth to increase annual wellness visits for screening promotion. While significant delays persist for scheduling colonoscopies, clinics were encouraged to promote stool-based tests as a primary screening modality for average-risk patients.
\end{abstract}

Keywords Implementation science $\cdot$ Formative research $\cdot$ COVID-19 $\cdot$ Rural health $\cdot$ Colorectal cancer

The Appalachian region of Kentucky is part of a four-state, 107-county colorectal cancer (CRC) "hotspot" cluster where $\mathrm{CRC}$ death rates are significantly higher than elsewhere in the USA [1]. Sixty of the 107 counties are located in central and eastern Kentucky, and of those, 54 are designated as Appalachian by the Appalachian Regional Commission [2].

Aaron J. Kruse-Diehr

kruse-diehr@uky.edu

1 University of Kentucky College of Public Health, 151 Washington Avenue, Bowman Hall 345, Lexington, KY 40536, USA

2 Markey Cancer Center, Lexington, KY, USA

3 University of Kentucky College of Medicine, Prevention Research Center, Lexington, KY, USA

4 Northeast Kentucky Area Health Education Center, Morehead, KY, USA
Like elsewhere in Appalachia, these areas of Kentucky are characterized by high levels of rurality, lower income levels, higher rates of unemployment, lower education levels, and generally poorer health outcomes [3]. In addition to increased CRC mortality, CRC incidence rates are higher throughout Appalachia, particularly in Kentucky where some of the highest incidence and mortality rates in the USA are located $[4,5]$. The many socioecological disparities in the region pose unique challenges to researchers seeking to improve CRC outcomes. To maximize effectiveness of interventions, researchers must implement multiple approaches in collaboration with trusted community partners.

Previous research has showcased the effectiveness of establishing academic partnerships with primary care clinics and federally qualified health centers (FQHCs) in improving $\mathrm{CRC}$ screening outcomes in rural areas [6-8]. FQHCs serve populations in underserved areas by providing primary care and preventive services to persons across the lifespan, regardless 
of their ability to pay [9]. These organizations are communitybased, nonprofit, or public and are governed by a board of directors composed of citizens or patients from their service area. Kentucky has nearly 350 clinics [10] designated as FQHCs due to receipt of grant funding from the Health Resources and Services Administration Bureau of Primary Care and specific reimbursement from Medicare and Medicaid [11]. In Appalachian Kentucky, many individuals receive their medical care from these rural primary care clinics and FQHCs [12]. Because of the lower number of physicians practicing in Appalachian Kentucky, clinics also employ physician extenders such as advance practice registered nurses (APRN), physician assistants (PA), and certified medical assistants (CMA) to assist with providing care. These medical staff often live and work within their own communities, creating opportunities to develop trust and rapport with their patients. Combined with their medical training, the aforementioned characteristics make these physician extenders ideal partners to develop and deliver locally tailored health interventions.

Researchers at the University of Kentucky (UK) Markey Cancer Center (MCC) have a long and robust history of collaboration with primary healthcare providers and clinics, area health education centers (AHECs), and communities across rural Appalachian Kentucky. Some recent projects include the adaptation of the proactive office encounter model to identify gaps in preventive care in an eight-site FQHC [13]; a collaboration with 66 rural practices to increase CRC screening [14]; the development of the Appalachian Community Cancer Network through which a physical activity and screening intervention to reduce regional cancer burden was implemented [15]; and a 5-year multisite, multilevel intervention in Appalachian Kentucky and Ohio to increase CRC screening and follow-up care, as part of the Accelerating Colorectal Cancer Screening and Follow-up through Implementation Science (ACCSIS) National Cancer Institute-funded Cancer Moonshot ${ }^{\mathrm{SM}}$ [16]. Collectively, these and other similar projects have helped establish partnerships with community providers, develop trusting relationships with patients, and ultimately improve health outcomes within Appalachian Kentucky communities.

In 2018, the National Cancer Institute called for research initiatives specifically targeting the development of research capacity in clinics that serve underserved rural populations [17]. Researchers at MCC partnered with the Northeast Kentucky AHEC and responded to the initiative proposing to work with four primary care clinics in Appalachian Kentucky. Three objectives were proposed: (1) assessment of clinic capacity for data collection, compilation, and analysis; (2) identification of facilitators and barriers to clinic implementation of evidence-based interventions (EBIs); and (3) pilot testing of individually tailored, multilevel EBIs that address CRC screening and follow-up. Each of these objectives was selected to strengthen clinic capacity to participate in future larger scale multilevel cancer control interventions. However, a set of unexpected barriers arose from the spread of COVID-19, a pandemic that significantly altered the form and function of primary care clinics serving Appalachian Kentucky. This paper describes the formative process of building and maintaining the research capacity of this initiative with providers and staff in the four partner clinics, and the necessary adaptations that were selected collaboratively in response to statewide and clinic-specific COVID-19 restrictions.

\section{Methods}

All proposed project activities were approved by the UK Institutional Review Board and the UK MCC Protocol Review and Monitoring Committee. The project work plan (Table 1) includes a series of steps to be carried out over the one-year project duration. Through the partnership with Northeast Kentucky AHEC, primary care clinics were identified and invited to participate in the project. One of AHEC's main responsibilities is facilitating community rotations for medical, nursing, and other allied health students, which requires extensive interactions with primary care clinics throughout its 17-county service area. The selection process included developing a list of potential clinics and reviewing data on clinic catchment areas, patient population, volume, and participation in other research projects. At the time of the selection process, there were several CRC screening projects ongoing in the study area within primary care clinics, so it was necessary to catalog ongoing projects to avoid potentially skewing the results of those other concurrent studies. At the same time primary care clinics were being recruited, a community advisory board (CAB) was developed. The $\mathrm{CAB}$ provided community perspectives to help keep the project grounded in terms of local needs, interests, and values.

Table 1 Project workplan by quarters

\begin{tabular}{lllll}
\hline Project activity & Q1 & Q2 & Q3 & Q4 \\
\hline IRB approval & X & & & \\
Primary care clinic recruitment & X & & & \\
CAB meetings & X & X & X & \\
Key informant interviews & X & X & & \\
Interview transcription and analyses & X & X & & \\
Clinic selection & & $X$ & & \\
Collection of clinic EHR data & & $X$ & $X$ & \\
Clinic intervention implementation & & & $X$ & X \\
Promotion of clinics as research partners & & & & X \\
Project evaluation & $\mathrm{X}$ & $\mathrm{X}$ & $\mathrm{X}$ & $\mathrm{X}$ \\
\hline
\end{tabular}


Based on self-selection and having met necessary inclusion criteria, ultimately, four primary care clinics agreed to participate in the project after which formative evaluation activities began.

The first formative evaluation step was to conduct key informant interviews and collect data to provide in-depth information about clinic operations in the context of EBIs related to CRC screening, including identifying existing clinic assets and barriers - both actual and perceived-to implementing those EBIs. The small number of physicians in the FQHCs made it critical to include multiple medical professionals (MD, DO, APRN, PA, CMA) in key informant interviews, as each of these individuals takes an active role in promoting CRC screening in their respective clinic patient populations. A total of seven individuals, primarily serving in nursing roles in their respective clinics, agreed to participate. Two members of the research team conducted phone interviews using a researcher-designed, semi-structured interview guide. The guide included questions to explore clinicwide CRC screening practices and probe further to uncover specific patient-, provider-, and clinic-side barriers to CRC screening. Each interview lasted between 30 and $45 \mathrm{~min}$, and responses were recorded, transcribed verbatim, and separately coded by two independent researchers for overarching themes and sub-themes using both deductive and inductive approaches. Few coding discrepancies arose and were subsequently resolved via investigator debriefings.

Drawing from previous experience with CRC screening and follow-up in Appalachian Kentucky clinics, clinic champions were identified for each clinic, and a series of meetings was scheduled with clinic champions and managers to introduce evidence-based multilevel intervention strategies selected from the Community Guide [18], multiple literature reviews, and the research team's previous work, as well as to assist clinic personnel with selecting approaches that could be best tailored to their clinic populations. For example, at the patient level, we presented options such as mailed fecal immunochemical tests (FIT) [19], improved and expanded patient education materials showcasing different screening options beyond colonoscopy [20], and patient reminders (such as annual wellness visit birthday card reminders, phone calls, or mailings) [21]. At the provider level, options included provider assessment and feedback [22], provider education, and provider reminders [21] to encourage patients to undergo screening. Finally, at the clinic level, we introduced strategies such as the adaptation of electronic health record (EHR) systems to better capture screening and follow-up results; clinic-wide promotion of annual wellness visits, during which providers would likely have more time to promote CRC screening [23, 24]; and implementation of automated EHR alert systems [21] to remind nurses and providers of overdue screenings.

\section{Results}

\section{Key Informant Interview Themes}

Seven key informant interviews were conducted across the four participating primary care clinics. Emergent themes from the analysis of the key informant interviews found barriers at patient, provider, and clinic levels. At the patient level, the most frequently cited barriers included fatalism related to surviving cancer and local residents not wanting to find out if they have cancer. One provider described her patients' concerns in the following manner: "You don't need to go digging around for things." Another provider described this mindset as part of the local culture, commenting, "[They believe] if it ain't broke, don't fix it." Other identified patient barriers included fear of various negative outcomes, such as bowel perforation, chemotherapy, death, and a perception that the treatment for CRC is worse than the diagnosis. Providers also noted that patients often have competing medical priorities that tend to minimize their concern for CRC screening: "Sometimes they think, 'Well maybe I should get checked for lung cancer,' but I don't think colon cancer is at their forethought." Furthermore, it was noted that prevention in general is often not a priority for patients, one provider commenting, "They just want to talk about what they're here for generally [ ... ] I mean, when people come in for a sore throat, they don't want to talk about their bowels." The other most common patient-side barriers included financial/ insurance concerns, lack of knowledge and education (including illiteracy), and distance-related issues for rural-living patients (including transportation concerns).

Numerous other perceived and actual barriers were found at provider and clinic levels. Perhaps the most salient concern was in regard to EHR capacities and limitations related to CRC screening and follow-up tracking. Although providers said their EHR systems were successful at keeping track of unscreened patients, with out-of-system referrals, they encountered difficulty tracking patient follow through with scheduled colonoscopies, thus creating potential gaps in care. Furthermore, providers almost universally identified varying levels of limitations in terms of what can be added to EHR platforms. For example, interviewees noted that it would likely be impossible to keep track of the number of people provided with educational materials using their EHR systems and that they would have those types of implementation outcomes in a separate spreadsheet. Furthermore, while clinic personnel were willing to share de-identified data from their EHRs, in many instances, they lacked the technical assistance and/or infrastructure to provide such data. Other provider barriers included time and workload concerns, concerns about the types or availability of educational materials, and the differing methods of follow-up with patients (i.e., some are contacted via phone whereas others are reached via mail). Lastly, 
providers identified personal preferences for screening modalities, many preferring colonoscopies to stool-based testing. One individual commented, "We usually offer the colonoscopy first because it's the best, and then if we [can't] get anywhere with ... educating them ... we go ahead and offer the Cologuard." Similarly, when asked about the availability of FIT kits, another provider said, "We can get that to anyone who's not willing to get a colonoscopy." In other words, clinics indicated they were offering stool-based test options only to those who first refused colonoscopy.

\section{Proposed Project Activities}

Collectively, the findings from these key informant interviews provided a solid foundation for the remaining activities of the project. However, the increase in risk of COVID-19 infections caused a series of changes in the daily operations of the primary care clinics at the geographic, clinic, provider, and patient levels. Due to state mandate, hospitals across Kentucky paused elective procedures, including colonoscopies, resulting in clinics ceasing referrals. The pause in elective procedures also necessitated that many hospitals in Appalachian Kentucky reduce their staff through furloughs and layoffs. Many clinics changed their hours of operation and transitioned to a hybrid model in which most patients were seen virtually through telehealth, whereas other clinics closed altogether for several weeks. Clinics that remained open did not always continue recommending CRC screening at the same rate as before the pandemic due to time concerns and patients' competing priorities. As a result, the investigators changed their approach to working with the clinics and suggested that additional discussions were needed to explore possible adaptations to strategies previously introduced to clinics.

After discussions with clinic managers and staff, a collaborative decision was made to pause the project temporarily as investigators and clinic staff accommodated changing priorities and resource allocation due to the COVID-19 pandemic, during which time clinics reduced their focus on screening and experienced significantly lower patient volumes. When investigators and clinic staff agreed it was appropriate and feasible to re-engage in project activities, new priorities emerged based on residual complications from the pandemic, impacting the priority EBIs for clinics. Due to ongoing backlog for scheduling colonoscopies at local/regional endoscopy centers, clinic champions were more open to increasing mailed, homebased stool-based testing efforts as a screening option. The FIT-DNA test (i.e., Cologuard $囚$ ) was prioritized over FIT as a stool-based option due to Cologuard $\AA$ requiring fewer clinic resources than FIT. Clinic champions also prioritized provider education, reminders, and assessment and feedback EBIs, as provider screening orders had been greatly reduced over the preceding months due to COVID-19, and they were looking for innovative methods by which they could increase screening recommendations. Additionally, due to the decrease in in-person patient visits, clinic champions were interested in exploring novel interventions to increase annual wellness visits to assist in recovery of CRC screening rates. On the whole, clinics were eager to consider any EBI that could help elevate screening to pre-pandemic levels. At the same time, EBIs involving patient- or provider-oriented printed materials were deemed less appropriate by clinics, with increased focus on digital materials to reduce the need for personal contact in handling physical materials. Changes in clinic screening priorities are summarized in Table 2 .

These shifting priorities led investigators to invest in more discussion with clinics about how to change from a "colonoscopy first" model to a shared decision-making model in which patients could choose at-home stool-based options if they were not comfortable with colonoscopy wait times or COVID testing requirements prior to colonoscopy. These discussions were guided by National Colorectal Cancer Roundtable guidelines on reigniting CRC screening during COVID-19 [25] and the need to prioritize colonoscopy scheduling for those with abnormal stool tests or other high-risk patient groups.

\section{Discussion}

The COVID-19 pandemic resulted in an immediate impact on primary care practices, particularly in rural areas like Appalachian Kentucky. The resulting changes in daily practice and workflow impacted CRC screening immediately and are expected to negatively influence CRC outcomes for a sustained period of time. Nationwide, from April 2019 to April 2020, the number of colonoscopies and biopsies was reduced by $90 \%$, resulting in 1.7 million missed colonoscopies and nearly 19,000 missed or delayed CRC diagnoses between March and June 2020 [25-27]. These delays in care - combined with reduced access due to temporary closures of primary care clinics, healthcare practitioner furloughs, and pauses in elective or specialty care - are expected to result in over 4500 excess CRC deaths across the next decade [28]. Given that rural areas of the USA, in general, are disproportionately burdened by higher incidence and mortality rates for many preventable cancers [29], it is likely that residents of Appalachian Kentucky will bear a substantial proportion of the cancer burden related to COVID-19 and its sequelae.

One common method used by rural providers to ensure continuity of care is telehealth, as was the case with the clinics in our study. With in-person visits being 30-50\% less frequent than pre-COVID-19, telehealth is an encouraged and recommended method of care for appropriate visit types, such as 
Table 2 Clinic interest in selected EBIs before and during COVID-19

\begin{tabular}{|c|c|c|}
\hline EBI & Pre-COVID & During COVID \\
\hline *Patient reminder letters & Limited use & Increased interest-to increase patient volume/CRC screening orders \\
\hline Patient education & Focus on print materials & Digital and social media only \\
\hline *Provider assessment/feedback & Limited & Increased interest-to motivate providers to increase screening orders \\
\hline *Annual wellness visit promotion & Important but not emphasized & Higher emphasis as strategy to increase patient volume \\
\hline Patient navigation & Interested but resources limited & Not interested-no staff resources available \\
\hline FIT/Cologuard & Not priority; emphasis on colonoscopy & $\begin{array}{l}\text { Open to at-home stool-based testing; especially Cologuard } \\
\text { because of more resource support }\end{array}$ \\
\hline *Provider education & General interest & $\begin{array}{l}\text { Greater interest in education on communication methods to } \\
\text { motivate patients to get screened (due to loss of patient } \\
\text { volume due to COVID) }\end{array}$ \\
\hline
\end{tabular}

*Strategy ultimately chosen by clinics for implementation

annual wellness visits during which clinicians might normally provide routine screening recommendations [30]. Factors such as the Centers for Medicare \& Medicaid Services expanding Medicare telehealth benefits and the Department of Health and Human Services allowing greater flexibility for physicians to see patients using telehealth methods have further supported its use [31]. Nevertheless, barriers persist for providers who use telehealth, including requirements for providers to be licensed in the same state where the patient is located and concerns related to technology [32]. These issues are particularly salient in rural areas where broadband access might be unreliable or unavailable, physical travel is often problematic, or patients may have to travel across state borders to access healthcare - areas of concern to many residents of Appalachian Kentucky. Furthermore, despite nationwide office visit volume remaining low, telehealth use has also decreased to pre-pandemic levels [33], leading to probable gaps in patient care that are likely to disproportionately affect rural areas. Clearly, to maintain continuity of care and avoid increased cancer mortality rates - particularly for screenable cancers such as CRC - there is a need for innovative policies and solutions.

To reduce the burden of excess CRC mortality in Appalachian Kentucky, there are a variety of options available to clinicians, including the use of at-home stool-based tests like fecal occult blood tests (FOBT), FIT, and FIT-DNA for average-risk patients. The use of stool-based tests as a firstline approach for CRC screening is especially important when elective procedures like colonoscopies are either unavailable or backlogged, as is the case in many rural communities. Due to these backlogs, high-risk patients or those with abnormal FIT/FOBT should be given highest patient priority to receive colonoscopies [34]. For research partners working with clinic partners in Appalachia, it is critical they be particularly understanding of clinic and regional challenges to addressing CRC screening and adjust their approaches accordingly [35], including providing additional flexibility on timelines and project activities as needed. Given that adaptations are common when integrating EBIs into regular practice [36], researchers should welcome necessary clinic EBI adaptations and evaluate how selected adaptations affect implementation success and organizational capacity [37]. One method to promote successful implementation during rapidly changing situations is by adopting a participatory implementation science approach to promote constant clinic-researcher engagement and allow clinic partners to take the lead in selecting and adapting EBIs they can confidently implement [38]. Members of the academic research team, then, can provide necessary technical assistance and help with tracking adaptations to reduce clinic burden.

In this project, clinic staff expressed specific concern with how to continue CRC screening during COVID-19, and they requested additional training to address this concern. One excellent reference for clinicians is the National Colorectal Cancer Roundtable's playbook for reigniting CRC screening during COVID-19 [25], a document that, among providing many other suggestions, promotes the prioritization of stoolbased screening for patients at average risk for CRC. In our key informant interviews, clinicians noted a preference for colonoscopy and stated they normally only suggested a stool-based test after patient refusal of colonoscopy. While elective procedures remain backlogged, however, research partners should emphasize the need to prioritize stool-based $\mathrm{CRC}$ screening for average-risk populations. One way to do so is to share research on FIT and other stool-based tests with clinic partners. For example, one study discovered that patients were more likely to be screened by FIT (34.2\%) than colonoscopy $(24.6 \%)$, while an another noted that $69 \%$ of patients who received a choice of stool-based test or colonoscopy were screened, compared to only $38 \%$ who were only offered colonoscopy [20,39]. Given that studies suggest patient preference for stool-based CRC screening, additional 
research is needed to fully understand provider-side barriers to recommending these tests.

\section{Conclusion}

Perhaps the most salient lesson from this formative project is confirmation of the need for both flexibility and adaptability when working with primary care clinics in Appalachian Kentucky. Flexibility is required in adjusting project plans to current events like COVID-19 and in adapting intervention strategies to the "new normal." Another lesson from this project relates to EHR concerns, as our rural clinics often lacked the infrastructure to produce important data to track proposed project outcomes, such as follow-up of out-of-system colonoscopies. Many rural clinics do not have in-house information technology support and instead rely on consultants. Such situations mean that requests for data require additional expenditures and take more time than would be required with inhouse IT support. Researchers can reduce this barrier by focusing on measurement tools that can be completed easily and will not be burdensome to clinic staff. Finally, in the context of COVID-19, researchers need to consider that clinics are already overburdened and are likely to be even more burdened due to reduced hours, staff furloughs, and in some cases with temporary closing. Given that the effects of COVID-19 are likely to be felt in Appalachian Kentucky for many years to come, researchers should be encouraged to adopt empathetic, flexible, and clinic-driven approaches in their future partnerships.

Acknowledgments The authorship team would like to acknowledge our primary care clinic partners in this study: St. Claire Family MedicineMorehead, St. Claire Family Medicine-Sandy Hook, PrimaryPlus-South Shore, PrimaryPlus-Flemingsburg

Availability of Data and Material Available upon request. Code Availability Not applicable.

Authors' Contribution All authors contributed to the study conception and design. Material preparation, data collection and analysis were performed by AK-D, MD, MC, AC, MR, DG, and SR. The first draft of the manuscript was written by AK-D, MD, MC, and AC. All authors commented on previous versions of the manuscript and contributed to revisions as needed. All authors read and approved the final manuscript.

Funding This study was funded by an administrative supplement to the Markey Cancer Center NCI Cancer Center Support Grant and was supported by the Markey Cancer Center Shared Resource Facilities (P30CA177558, Evers: PI).

\section{Declarations}

Conflict of interest The authors declare that they have no conflict of interest.

\section{References}

1. Siegel RL, Sahar L, Robbins A, Jemal A (2015) Where can colorectal cancer screening interventions have the most impact? Cancer Epidemiol Biomarkers Prev 24(8):1151-1156

2. Appalachian Regional Commission (n.d.) About the Appalachian region. https://www.arc.gov/about-the-appalachian-region/ (accessed Oct 8, 2020)

3. Appalachian Regional Development Act Amendments of 2007 (2007) Rep. Oberstar JLD-M-, trans. H.R.799. 110th ed

4. Wilson RJ, Ryerson AB, Singh SD, King JB (2016) Cancer incidence in Appalachia, 2004-2011. Cancer Epidemiol Biomarkers Prev 25(2):250-258

5. US Cancer Statistics Working Group (2017) United States Cancer Statistics: 1999 - 2014 Incidence and mortality web-based report. US Department of Health and Human Services, Atlanta GA

6. Arnold CL, Rademaker A, Liu D, Davis TC (2017) Changes in colorectal cancer screening knowledge, behavior, beliefs, self-efficacy, and barriers among community health clinic patients after a health literacy intervention. J Community Med Healthl Educ 7(1): 497

7. Arnold CL, Rademaker A, Wolf MS, Liu D, Hancock J, Davis TC (2016) Third annual fecal occult blood testing in community health clinics. Am J Health Behav 40(3):302-309

8. Honeycutt S, Green R, Ballard D, Hermstad A, Brueder A, Haardörfer R, Yam J, Arriola KJ (2013) Evaluation of a patient navigation program to promote colorectal cancer screening in rural Georgia, USA. Cancer 119(16):3059-3066

9. Kentucky Primary Care Association (n.d.) What are federally qualified health centers (FQHCs)? https://kpca.net/federally-qualifiedhealth-centers (accessed June 16, 2020)

10. NPIdb (n.d.) Federally qualified health center. https://npidb.org/ organizations/ambulatory_health_care/federally-qualified-healthcenter-fqhe 261qf0400x/ (accessed June 16, 2020)

11. National Association of Community Health Centers (n.d.) Federally qualified health centers. http://www.nachc.org (accessed June 16, 2020)

12. Phillips RL, Jr., Klink K, Petterson SM, KoJima N, Bazemore AW (2014) The continued importance of small practices in the primary care landscape. Am Fam Physician 90(4). https://www.aafp.org/ afp/2014/0815/od3.html (accessed June 16, 2020)

13. Vanderpool RC, Moore SC, Stradtman LR, Carman AL, Kurgat HL, Fain P (2016) Adaptation of an evidence-based intervention to improve preventive care practices in a federally qualified health center in Appalachian Kentucky. J Health Care Poor Underserved 27(4A):46-52

14. Dignan M, Shelton B, Slone SA, Tolle C, Mohammad S, Schoenberg N, Pearce K, van Meter E, Ely G (2014) Effectiveness of a primary care practice intervention for increasing colorectal cancer screening in Appalachian Kentucky. Prev Med 58:70-74

15. Baltic RD, Weier RC, Katz ML, Kennedy SK, Lengerich EJ, Lesko SM, Reese D, Roberto KA, Schoenberg NE, Young GS, Dignan MB, Paskett ED (2015) Study design, intervention, and baseline characteristics of a group randomized trial involving a faith-based healthy eating and physical activity intervention (Walk by Faith) to reduce weight and cancer risk among overweight and obese Appalachian adults. Contemp Clin Trials 44:1-10

16. National Cancer Institute (2020) Accelerating colorectal cancer screening and follow-up through implementation science (ACCSIS). https://healthcaredelivery.cancer.gov/accsis/ (accessed June 16, 2020)

17. National Cancer Institute (2020) Rural cancer control. https:// cancercontrol.cancer.gov/research-emphasis/rural-cancer-control (accessed Oct 8, 2020) 
18. The Community Guide (n.d.) Cancer. https://www. thecommunityguide.org/topic/cancer (accessed Dec 2, 2020)

19. Kemper KE, Glaze BL, Eastman CL, Waldron RC, Hoover S, Flagg T'R, Tangka FKL, Subramanian S (2018) Effectiveness and cost of multilayered colorectal cancer screening promotion interventions at federally qualified health centers in Washingston state. Cancer 124(21):4121-4129

20. Inadomi JM, Vijan S, Janz NK, Fagerlin A, Thomas JP, Lin YV, Muñoz R, Lau C, Somsouk M, el-Nachef N, Hayward RA (2012) Adherence to colorectal cancer screening: a randomized clinical trial of competing strategies. Arch Intern Med 172(7):575-582

21. Sequist TD, Zaslavsky AM, Marshall R, Fletcher RH, Ayanian JZ (2009) Patient and physician reminders to promote colorectal cancer screening: a randomized controlled trial. Arch Intern Med 169(4):364-371

22. The Community Guide (n.d.) Cancer screening: provider assessment and feedback - colorectal cancer. https://www. thecommunityguide.org/findings/cancer-screening-providerassessment-and-feedback-colorectal-cancer (accessed Dec 2, 2020)

23. Jiang M, Hughes DR, Wang W (2018) The effect of Medicare's annual wellness visit on preventive care for the elderly. Prev Med 116:126-133

24. Camacho F, Yao N, Anderson R (2017) The effectiveness of Medicare wellness visits in accessing preventive screening. J Prim Care Community Health 8(4):247-255

25. National Colorectal Cancer Roundtable (2020) Reigniting colorectal cancer screening as communities face and respond to the COVID-19 pandemic: a playbook. https://nccrt.org/resource/aplaybook-for-reigniting-colorectal-cancer-screening-ascommunities-respond-to-the-covid-19-pandemic/ (accessed Sept 22, 2020)

26. Komodo Health, Fight Colorectal Cancer (2020) New colorectal cancer diagnoses fall by one-third as colonoscopy screenings and and biopsies grind to a halt during height of COVID-19. https:// fightcolorectalcancer.org/wp-content/uploads/2020/05/COVID19Impact-on-CRC-Patients_Research-Brief_Komodo-Health-FightCRC.pdf (accessed Sept 22, 2020)

27. IQVIA Institute (2020) Shifts in healthcare demand, delivery, and care during the COVID-19 era. https://www.iqvia.com/insights/theiqvia-institute/covid-19/shifts-in-healthcare-demand-delivery-andcare-during-the-covid-19-era (accessed Sept 22, 2020)

28. Sharpless NE (2020) COVID-19 and cancer. Science 368(6497): 1290

29. National Advisory Committee on Rural Health and Human Services (2019) Examining rural cancer prevention and control efforts: policy brief and recommendations to the secretary. https:// www.hrsa.gov/sites/default/files/hrsa/advisory-committees/rural/ publications/2019-Cancer-Control.pdf (accessed Sept 22, 2020)
30. The Larry A. Green Center (2020) Quick COVID-19 primary care survey: series 20. https://www.green-center.org/covid-survey (accessed Sept 22, 2020)

31. Kutikov A, Weinberg DS, Edelman MJ, Horwitz EM, Uzzo RG, Fisher RI (2020) A war on two fronts: cancer care in the time of COVID-19. Ann Intern Med 172(11):756-758

32. Sodhi M (2020) Telehealth policies impacting federally qualified health centers in face of COVID-19. J Rural Health. 37:158-160. https://doi.org/10.1111/jrh.12445

33. Fox B, Sizemore JO (2020) Telehealth: fad or the future. Epic Health Research Network. https://ehrn.org/wp-content/uploads/ telehealth-fad-future.pdf (accessed Sept 22, 2020)

34. Balzora S, Issaka RB, Anyane-Yeboa A, Gray DM 2nd, May FP (2020) Impact of COVID-19 on colorectal cancer disparities and the way forward. Gastrointest Endosc 92(4):946-950

35. Nodora JN, Gupta S, Howard N, Motadel K, Propst T, Rodriguez J, Schultz J, Velasquez S, Castañeda SF, Rabin B, Martínez ME (2020) The COVID-19 pandemic: identifying adaptive solutions for colorectal cancer screening in underserved communities. J Natl Cancer Inst. https://doi.org/10.1092/jnci/djaa117

36. Cohen DJ, Crabtree BJ, Etz RS et al (2008) Fidelity versus flexibility: translating evidence-based research into practice. Am J Prev Med 35(5 Suppl):S381-S389

37. Balasubramanian BA, Cohen DJ, Davis MM et al (2015) Learning evaluation: blending quality improvement and implementation research methods to study heatlhcare innovations. Imp Sci 10:31

38. Ramanadhan S, Davis MM, Armstrong R, Baquero B, Ko LK, Leng JC, Salloum RG, Vaughn NA, Brownson RC (2018) Participatory implementation science to increase the impact of evidence-based cancer prevention and control. Cancer Causes Control 29(3):363-369

39. Quintero E, Castells A, Bujanda L, Cubiella J, Salas D, Lanas Á, Andreu M, Carballo F, Morillas JD, Hernández C, Jover R, Montalvo I, Arenas J, Laredo E, Hernández V, Iglesias F, Cid E, Zubizarreta R, Sala T, Ponce M, Andrés M, Teruel G, Peris A, Roncales MP, Polo-Tomás M, Bessa X, Ferrer-Armengou O, Grau J, Serradesanferm A, Ono A, Cruzado J, Pérez-Riquelme F, Alonso-Abreu I, de la Vega-Prieto M, Reyes-Melian JM, Cacho G, Díaz-Tasende J, Herreros-de-Tejada A, Poves C, Santander C, González-Navarro A (2012) Colonoscopy versus fecal immunochemical testing in colorectal-cancer screening. N Engl J Med 366(8):697-706

Publisher's Note Springer Nature remains neutral with regard to jurisdictional claims in published maps and institutional affiliations. 